\title{
White Matter Integrity in the Right Hemisphere Correlates with the Reading Span
}

\author{
Ekaterina V. Pechenkova ${ }^{1}$, Yana R. Panikratova ${ }^{2}$, Maria A. Fomina ${ }^{3}$, Elena A. Mershina ${ }^{4}$, \\ Daria A. Bazhenova ${ }^{4}$, Liudmila A. Makovskaya ${ }^{5}$, Alena D. Rumshiskaya ${ }^{6}$, Olga V. \\ Fedorova $^{7}$, Valentin E. Sinitsyn ${ }^{4}$, Irina S. Lebedeva ${ }^{2}$, Roza M. Vlasova ${ }^{8}$
}

\footnotetext{
${ }^{1}$ Laboratory for Cognitive Research, Higher School of Economics, Moscow, Russia Correspondence: 4 Armyanskiy ln., bld. 2, 101000 Moscow Russia; evp@ virtualcoglab.org ${ }^{2}$ Laboratory of Neuroimaging and Multimodal Analysis, Mental Health Research Center, Moscow, Russia

${ }^{3}$ Faculty of Psychology, Lomonosov Moscow State University, Moscow, Russia

${ }^{4}$ Medical Research and Educational Center (Lomonosov University Clinic), Moscow, Russia

${ }^{5}$ Radiology Department, Federal Center of Treatment and Rehabilitation, Moscow, Russia

${ }^{6}$ Radiology Department, Davidovskiy City Clinical Hospital, Moscow, Russia

${ }^{7}$ Faculty of Philology, Lomonosov Moscow State University, Moscow, Russia

${ }^{8}$ Department of Psychiatry, University of North Carolina at Chapel Hill, USA
}

\begin{abstract}
Although working memory (WM) is crucial for intellectual abilities, not much is known about its brain underpinnings, especially the structural connectivity. We used diffusion tensor imaging (DTI) to look across the whole brain for the white matter integrity correlates of the individual differences in the reading span (verbal WM capacity during reading) in healthy adults. Right-handed healthy native Russian speakers $(N=47)$ underwent DTI on a 3T Philips Ingenia scanner. Verbal WM was assessed with the Daneman-Carpenter reading span test (Russian version). Fractional anisotropy maps from each participant were entered into the group tract-based spatial statistics analysis with the reading span as a covariate; the results were TFCE-corrected. Reading span positively correlated with the white matter integrity in several sites of the right hemisphere: the body and the splenium of corpus callosum; the posterior limb of internal capsule; posterior corona radiata; and superior parietal white matter. Although the left hemisphere is central for verbal processing, we revealed the important role of the right hemisphere white matter for the verbal WM capacity. Our finding indicates that larger verbal working memory span may originate from additional processing resources of the right hemisphere.
\end{abstract}

Keywords: working memory, reading span, white matter, DTI 


\section{Introduction}

Working memory is a core process in the human cognitive and executive functions. It is crucial for learning and intellectual abilities, and working memory deficits are observed in many neurological conditions. However, not much is yet known about the brain systems underlying working memory, especially about the structural connectivity within these systems. The DTI studies of the WM correlates are handful, and they address different populations (from young children to ageing) and employ different measures of verbal and non-verbal working memory usually taken from the Wechsler Scale subtests (digit spans, sequencing, spatial span). Overall DTI data indicate that working memory implicates frontoparietal, posterior temporal, and precuneal white matter, as well as corpus callosum (Bathelt et al., 2017; Chung et al., 2018; Darki \& Klingberg, 2015; Klingberg, 2006; Olesen et al., 2003; Schulze et al., 2011). However, since working memory is a measure of both storage and information processing (Baddeley \& Hitch, 1974), the type of material and processing may play an important role. Therefore we focused on a specific measure of the working memory called reading span which characterizes the verbal working memory capacity during reading (Daneman, Carpenter, 1980). In the present study we used diffusion tensor imaging (DTI) to look across the whole brain for the white matter integrity correlates of the individual differences in the reading span in the healthy adult population.

\section{Methods}

\section{Participants}

Forty seven native speakers of Russian ( $N=47 ; 33$ females, aged $25 \pm 5$ y.o. $)$ who reported no neurological or mental conditions took part in the study. The project was approved by the Interuniversity Ethics Committee of Moscow. Participants gave written consent to the MRI and behavioral procedures and received financial compensation for their time and effort.

\section{Neuroimaging}

MR images were acquired on a 3T Philips Ingenia MRI scanner located at the Mental Health Research Center, Moscow, Russia. The DWI sequences had the following parameters: $\mathrm{b}=1000 \mathrm{~s} / \mathrm{mm}^{2}$, isotropic voxel size $2.5 \mathrm{~mm}, 32$ diffusion directions, AP phase encoding direction; $\mathrm{b}=0 \mathrm{~s} / \mathrm{mm}^{2}$ with both AP and PA phase encoding directions. The DTI data was 
complemented with the T1-weighted structural images acquired with the TFE sequence with parameters: $\mathrm{TR} / \mathrm{TE} / \mathrm{FA}=8 \mathrm{~ms} / 4 \mathrm{~ms} / 8^{\circ}, 170$ near-transverse slices, isotropic voxel size $1 \mathrm{~mm}$.

The data were processed with FSL software. Fractional anisothropy (FA) maps from each participant were entered into the tract-based spatial statistics (TBSS) analysis with the reading span as a covariate; the results were TFCE-corrected for multiple comparisons after 5000 permutations.

\section{Procedure}

The DWI was performed in either beginning or end of the one hour and a half neuroimaging session which included several experiments. The working memory assessment (reading span test) took place either before or after the scanning session. As a verbal working memory measurement we used the Russian version of the Daneman-Carpenter (Daneman, Carpenter, 1980) reading span test.

\section{Results}

Working memory capacity (reading span) positively correlated with the white matter integrity in several sites of the right hemisphere (according to the JHU white matter atlas): the body, the genu and the splenium of corpus callosum; the posterior limb of internal capsule; posterior thalamic radiation; posterior and anterior corona radiata; and superior parietal white matter (see Figure 1).

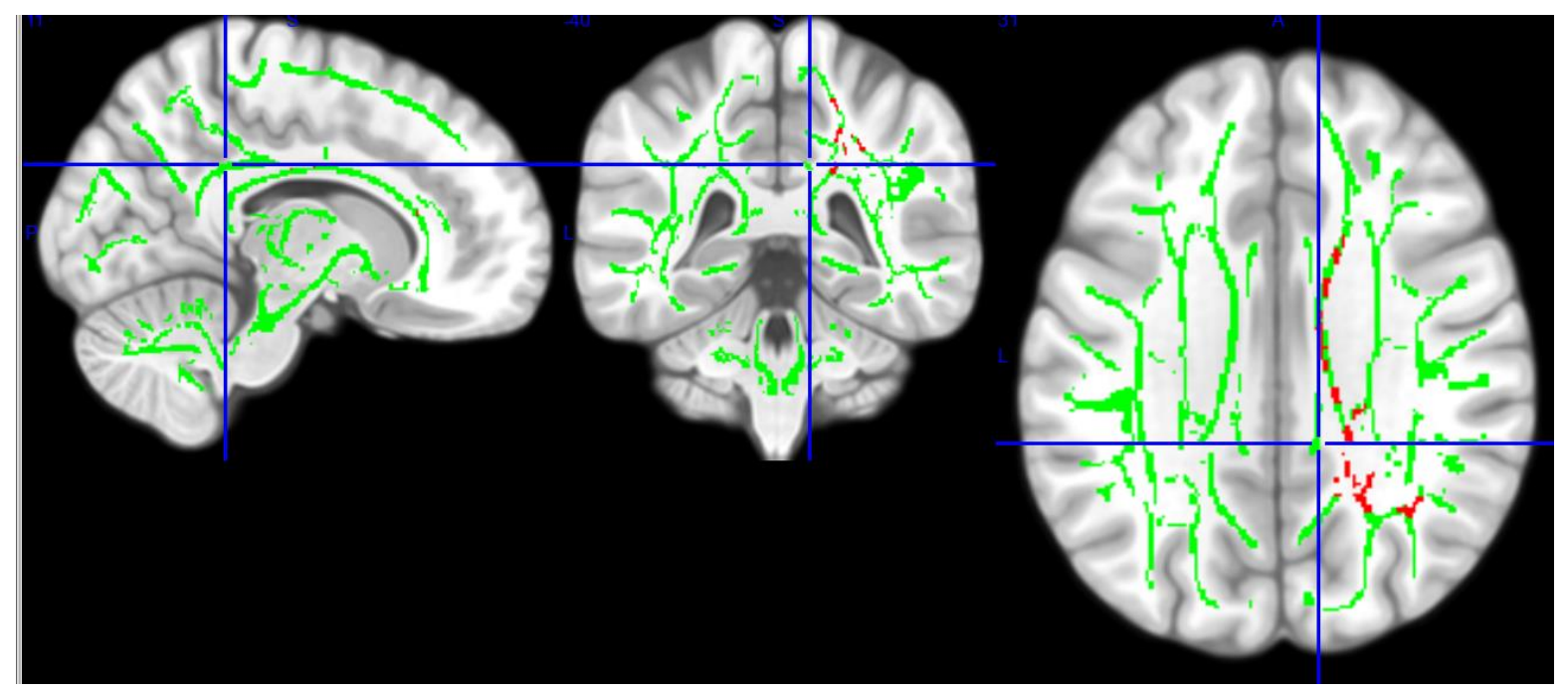

Figure 1. Portions of the tracts that demonstrated a positive correlation of the FA as a measure of the white matter integrity and the reading span (in read) overlaid over an FA skeleton (green) and MNI anatomical template. 


\section{Discussion}

Although the right hemisphere is often considered as nonverbal, the present study reveals the important role of the white matter of the right hemisphere for the verbal working memory capacity. These results are in general consistent with a handful of earlier DTI studies with children and ageing populations and different working memory measures (Chung et al., 2018). Our finding indicates that larger verbal working memory span may originate from additional processing resources of the right hemisphere. Looking for potential white-matter right-hemisphere correlates of the verbal working memory deficits seems to be a promising direction of future research.

\section{Acknowledgements}

The study was supported by RFBR grant 18-00-01598(18-00-01592). 


\section{References}

Baddeley, A. D., \& Hitch, G. (1974). Working memory. In Psychology of learning and motivation (Vol. 8, pp. 47-89). Academic press.

Bathelt, J., Gathercole, S. E., Johnson, A., \& Astle, D. E. (2018). Differences in brain morphology and working memory capacity across childhood. Developmental Science, 21(3), e12579.https://doi.org/10.1111/desc.12579

Chung, S., Fieremans, E., Kucukboyaci, N. E., Wang, X., Morton, C. J., Novikov, D. S., ... \& Lui, Y. W. (2018). Working memory and brain tissue microstructure: white matter tract integrity based on multi-shell diffusion MRI. Scientific reports, 8(1), 1-7. https://doi.org/10.1038/s41598-018-21428-4

Daneman, M., \& Carpenter, P. A. (1980). Individual differences in working memory and reading. Journal of Memory and Language, 19(4), 450.

Darki, F., \& Klingberg, T. (2015). The role of fronto-parietal and fronto-striatal networks in the development of working memory: a longitudinal study. Cerebral cortex, 25(6), 1587-1595.https://doi.org/10.1093/cercor/bht352

Klingberg, T. (2006). Development of a superior frontal-intraparietal network for visuo-spatial working memory. Neuropsychologia, 44(11), 2171-2177. https://doi.org/10.1016/j.neuropsychologia.2005.11.019

Olesen, P. J., Nagy, Z., Westerberg, H., \& Klingberg, T. (2003). Combined analysis of DTI and fMRI data reveals a joint maturation of white and grey matter in a fronto-parietal network. Cognitive Brain Research, 18(1), 48-57. https://doi.org/10.1016/j.cogbrainres.2003.09.003

Schulze, E. T., Geary, E. K., Susmaras, T. M., Paliga, J. T., Maki, P. M., \& Little, D. M. (2011). Anatomical correlates of age-related working memory declines. Journal of aging research, 2011. https://doi. org/10.4061/2011/606871 